\title{
A stochastic approach to the closure of accessible sets of control systems with application on homogeneous spaces
}

\section{Diego S. Ledesma \& Fabiano B. da Silva}

To cite this article: Diego S. Ledesma \& Fabiano B. da Silva (2018) A stochastic approach to the closure of accessible sets of control systems with application on homogeneous spaces, Dynamical Systems, 33:3, 519-535, DOI: 10.1080/14689367.2017.1397106

To link to this article: https://doi.org/10.1080/14689367.2017.1397106

Accepted author version posted online: 27

Oct 2017.

Published online: 11 Dec 2017.

Submit your article to this journal $₫$

Џ Article views: 32

View Crossmark data $\complement$ 


\title{
A stochastic approach to the closure of accessible sets of control systems with application on homogeneous spaces
}

\author{
Diego S. Ledesma a and Fabiano B. da Silva $\circledast^{b}$ \\ ${ }^{a}$ Departamento de Matemática, Universidade Estadual de Campinas, Campinas, Brazil; ${ }^{b}$ Departamento de \\ Matemática, Universidade Estadual Paulista, Bauru, Brazil
}

\begin{abstract}
Given a control system on a compact manifold $M$, we study conditions for the foliation defined by the accessible sets to be dense in $M$. For this, we relate the control system to a stochastic differential equation and, by the support theorem, we give a characterization of the density in terms of the infinitesimal generator of the diffusion and its invariant measures.
\end{abstract}

ARTICLE HISTORY

Received 21 March 2017

Accepted 23 October 2017

\section{KEYWORDS}

Control systems; diffusion processes; stochastic calculus; Brownian motion

\section{MATHEMATICS SUBJECT CLASSIFICATION CODES Primary: 60J60, 58E25; Secondary: 60J70, 37N35, $60 \mathrm{~J} 65,60 \mathrm{~J} 35$}

\section{Introduction}

A set of reachable points (accessible sets) by trajectories of the family of ordinary differential equations, parametrized by control parameters, have been studied not only in the dynamic aspect, but also in geometric and probabilistic terms. The geometric objects appear because accessible sets are intimately related to the group of transformations generated by the dynamical systems (see, for example, [1]), and probabilistic terms because there is a 'good approximation' between stochastic differential equations and control systems (see, for example, [2]).

The main setup is the following: consider, over a compact Riemannian manifold without boundary $(M, g)$, a family $F=\left\{X_{0}, X_{1}, \ldots, X_{k}\right\}$ of smooth vector fields over $M$ and the associated control system

$$
\dot{p}=X_{0}(p)+\sum_{i=1}^{k} u_{i}(t) X_{i}(p)
$$

with smooth functions $u_{i}: \mathbb{R}_{\geq 0} \rightarrow \mathbb{R}$. Denote by $p_{u}(t)$ the solution of (1) for some

$$
u=\left(u_{1}, \ldots, u_{k}\right) \in C^{\infty}\left(\mathbb{R}_{\geq 0}, \mathbb{R}^{k}\right),
$$

and such that $p_{u}(0)=p$. We call any such solution as a control path starting at $p$ and denote by $\mathcal{C P}$ the set of all control paths starting at $p$. 
Let $\mathcal{A}(p)$ be the accessible set from $p \in M$ defined by

$$
\mathcal{A}(p)=\left\{p_{u}(t) \in M, t \geq 0 \text { and } u \in C^{\infty}\left(\mathbb{R}_{\geq 0}, \mathbb{R}^{k}\right)\right\}
$$

(see [1] or [3] for more details). It is well known that the accessible sets determine a foliation with singularities (see, for example, [4]).

Our interest in this article is to find conditions that guarantee $\overline{\mathcal{A}(p)}=M$, for all $p \in M$. To do this, we will use the stochastic method. The motivation is the following. Let $\mathcal{W}(M)$ be the space of continuous paths $s:[0, \infty) \rightarrow M$ and $\mathcal{B}(\mathcal{W}(M))$ be the sigma algebra generated by the Borel cylinders sets. It is well known that associated to the control system (1), there exists a diffusion over $M$ with infinitesimal generator $\mathcal{L}$ given by

$$
\mathcal{L} f=\left(X_{0}+\frac{1}{2} \sum_{i=1}^{k} X_{i}^{2}\right) f
$$

(see, for example, [5]). Also, associated to the operator $\mathcal{L}$, there is a unique strongly Markovian system of probability measures $\left\{\mathbb{P}_{p}, p \in M\right\}$ over $\mathcal{W}(M)$ defined by

$$
\mathbb{P}_{p}[s(t) \in U]=P_{t}(p, U),
$$

for all Borel sets $U$ of $M$. Here, $P_{t}(p, \cdot)$ are the transition probabilities of the diffusion defined by $\mathcal{L}$. The measures $\mathbb{P}_{p}$ satisfy

(i) $\mathbb{P}_{p}[s \in \mathcal{W}(M), s(0)=p]=1$;

(ii) for all $f \in \mathcal{D}(\mathcal{L})$ and $p \in M$, we have that

$$
f(s(t))-f(0)-\int_{0}^{t}(\mathcal{L} f)(s(r)) d r
$$

is a $\left(\mathbb{P}_{p}, \mathcal{B}(\mathcal{W}(M))\right)$-martingale.

For more details, see [5, pp. 190-194].

The relations between the control system (1) and the SDE (2) are then given by the support theorem (see, for example, [5, Theorem 8.1, p. 431]) that states

$$
\operatorname{supp}\left(\mathbb{P}_{p}\right)=\overline{\mathcal{C P}},
$$

where $\operatorname{supp}\left(\mathbb{P}_{p}\right)$ is the smallest closed subset of $\mathcal{W}(M)$, with the condition $s(0)=p$, that carries probability $\mathbb{P}_{p}$ equal to 1 , and $\overline{\mathcal{C P}} \mathcal{P}_{p}$ is the closure of the set of all control paths starting at $p$. Therefore, the study of subsets of the control paths can be seen by examining the supports of transition probabilities, or, in the last instance, by supports of invariant measures given by the heat semigroup associated to these transition probabilities. Connections between accessible sets and supports of invariant measures have also been studied in the context of degenerate diffusion (see, for example, [6]).

With the above setup, the main purpose of our work is to determine when $\overline{\mathcal{C P}_{p}}=M$ or, as we mentioned before, when all invariant measures are supported in $M$. In particular, we will focus on control systems over homogeneous spaces. Using the approach introduced 
by Garnett [7] in the study of the harmonic measures for foliations (see also [8] and [9]), we will prove a condition that guarantees the density of accessible sets (see Theorem 5.2). We remark that the motive of working with foliations became natural because the control paths produce a foliation on the manifold. Therefore, our problem can be seen as the study of ergodicity of the foliation.

The article is organized as follows: In Section 2, we review the main tools of stochastic differential equations (SDEs) that are used in this study. In Section 3, we consider a diffusion, given by a Stratonovich SDE with the same smooth vector fields of (1), and characterize the closure of accessible set by transition probabilities and invariant measures for this SDE. In Section 4, we rewrite the previously mentioned result in the context of foliated spaces and give some examples as applications. We then applied, in Section 5, our results in a compact homogeneous manifolds under certain properties in the Lie algebras.

\section{Invariant measures for the stochastic differential equations}

The motivation of this section is to present an overview of the well-known facts from SDE theory, which will be used as tools for our main subject.

Given a family $F=\left\{X_{0}, X_{1}, \ldots, X_{k}\right\}$ of smooth vector fields in $M$ and a Brownian motion $B=\left(B_{1}, \ldots, B_{k}\right)$ in $\mathbb{R}^{k}$ based on a filtered probability space $(\Omega, \mathcal{F}, \mathbb{P})$, we consider the Stratonovich SDE

$$
\begin{aligned}
d p_{t} & =X_{0}\left(p_{t}\right) d t+\sum_{i=1}^{k} X_{i}\left(p_{t}\right) \circ d B_{t}^{i} \\
p_{0} & =p
\end{aligned}
$$

A solution for this equation is an adapted stochastic process $Y$ in $M$ satisfying

$$
\begin{aligned}
f\left(Y_{s}\right)-f(p) & =\int_{0}^{t} X_{0} f\left(Y_{s}\right) d t+\sum_{i=1}^{k} \int_{0}^{t} X_{i} f\left(Y_{s}\right) \circ d B_{s}^{i} \\
& =\sum_{i=1}^{k} \int_{0}^{t} X_{i} f\left(Y_{s}\right) d B_{s}^{i}+\int_{0}^{t}\left(X_{0}+\frac{1}{2} \sum_{i=1}^{k} X_{i}^{2}\right) f\left(Y_{s}\right) d s
\end{aligned}
$$

for all real-valued function $f \in C^{2}(M)$. In the integrals above, $d B_{s}^{i}$ and $\circ d B_{s}^{i}$ are used to denote Itô and Stratonovich integral, respectively.

When the manifold is compact, it is well known (see, for example, [10] or [5]) that there is a solution flow, i.e. a map $\varphi: \mathbb{R}_{\geq 0} \times M \times \Omega \rightarrow M$ such that

(i) $Y_{t}=\varphi(t, p, \cdot)$ solves the $\operatorname{SDE}(2)$ with $Y_{0}=p$;

(ii) for all $f$ in $C^{\infty}(M)$ and $\omega \in \Omega$, we have that $f(\varphi(t, \cdot, \omega))$ is a function in $C^{\infty}(M)$.

Associated to this solution flow, there are the transition probabilities $\left\{P_{t}(p, \cdot), p \in M, t \geq\right.$ $0\}$, given by the relation

$$
P_{t}(p, U)=\mathbb{E}\left[\chi_{U}(\varphi(t, p, \cdot)]=\mathbb{P}[\varphi(t, p, \cdot) \in U]=\left(\varphi(t, p, \cdot)_{*} \mathbb{P}\right)[U],\right.
$$


where $\chi_{U}$ is the characteristic function

$$
\chi_{U}(p)=\left\{\begin{array}{l}
1 \text { if } p \in U \\
0 \text { if } p \notin U
\end{array} .\right.
$$

If $\mathcal{B}(M)$ is the $\sigma$-algebra of all Borel sets of $M$, then $P_{t}: M \times \mathcal{B}(M) \rightarrow \mathbb{R}$ have the two following conditions (see [5, pp. 190-194]):

(a) for every $p \in M$, the map $P_{t}(p, \cdot): \mathcal{B}(M) \rightarrow \mathbb{R}$ is a probability measure;

(b) for every $U \in \mathcal{B}(M)$, the function $P_{t}(\cdot, U): M \rightarrow \mathbb{R}$ is Borel measurable.

Denote by $\varphi_{t}(x)=\varphi(t, x, \cdot): \Omega \rightarrow M$. The infinitesimal generator $\mathcal{L}$ is given by

$$
\mathcal{L} f(x)=\lim _{t \rightarrow 0} \frac{\mathbb{E}\left(f\left(\varphi_{t}(x)\right)\right)-f(x)}{t},
$$

where $\mathbb{E}\left(f\left(\varphi_{t}(x)\right)\right)$ is the expectation of the random variable $f\left(\varphi_{t}(x)\right): \Omega \rightarrow \mathbb{R}$ with respect to $\mathbb{P}$. Let $\mathcal{D}(\mathcal{L})$ be the set of continuous functions $f: M \rightarrow \mathbb{R}$ such that $\mathcal{L} f$ is continuous. It is well known that the relation between $\mathcal{L}$ and the coefficients of the SDE (2) is given by

$$
\mathcal{L} f=\left(X_{0}+\frac{1}{2} \sum_{i=1}^{k} X_{i}^{2}\right) f .
$$

Moreover, the transition probabilities generate a Markov semigroup $T_{t}: \mathcal{D}(\mathcal{L}) \rightarrow \mathcal{D}(\mathcal{L})$ $(t \geq 0)$ given by $T_{t}(f)(x)=\mathbb{E}\left(f\left(\varphi_{t}(x)\right)\right)$, whose infinitesimal generator is precisely $\mathcal{L}$. In fact, the relation between $T_{t}$ and $P_{t}$ can be seen by the induced measure theorem to the map $\varphi_{t}(x): \Omega \rightarrow M$, more precisely

$$
\begin{aligned}
T_{t}(f(x)) & =\mathbb{E}\left[f\left(\varphi_{t}(x)\right)\right] \\
& =\int_{\Omega} f\left(\varphi_{t}(x)\right) d \mathbb{P} \\
& =\int_{M} f(y) d\left(\left(\varphi_{t}(x)\right)_{*} \mathbb{P}\right) \\
& =\int_{M} f(y) P_{t}(x, d y) .
\end{aligned}
$$

And, as an implication of Dynkin's formula and Kolmogorov's backward equation, we have that

$$
\partial_{t}\left(T_{t} f\right)=T_{t}(\mathcal{L} f)=\mathcal{L}\left(T_{t} f\right)
$$

Since $M$ is compact, the space of measures on $M$ is the dual of the space of continuous functions on $M$. Therefore, a measure $\mu$ in $M$ is associated with the real-valued linear functional on $\mathcal{D}(\mathcal{L})$, and is given by

$$
f \mapsto \int_{M} f(x) d \mu(x)
$$


Because of the preceding remark, it makes sense to define the map $T_{t}^{*}$ on measure spaces by

$$
\left(T_{t}^{*} \mu\right)(f)=\int_{M}\left(T_{t} f\right)(x) \mu(d x) .
$$

Therefore, we say that $T_{t}^{*}$ preserves the measure $\mu$ (i.e. $T_{t}^{*} \mu=\mu$ ) if and only if

$$
\int_{M} T_{t} f(x) \mu(d x)=\int_{M} f(x) \mu(d x)
$$

for all $f \in \mathcal{D}(\mathcal{L})$. In this case, we say that $\mu$ is an invariant measure for the SDE (2).

The following result characterizes invariant measures in terms of infinitesimal generator $\mathcal{L}$, and it can be seen, for example, in [5, p. 277].

Proposition 2.1: $T_{t}^{*} \mu=\mu$ if and only if $\int_{M} \mathcal{L} f(x) \mu(d x)=0$ for all $f \in \mathcal{D}(\mathcal{L})$.

Proof: For all $f \in \mathcal{D}(\mathcal{L})$, by Equation (3), we obtain that

$$
\begin{aligned}
\left.\frac{d}{d t}\right|_{t=0} \int_{M} f(x)\left(T_{t}^{*} \mu\right)(d x) & =\left.\frac{d}{d t}\right|_{t=0} \int_{M} T_{t} f(x) \mu(d x) \\
& =\int_{M} T_{0}(\mathcal{L} f)(x) \mu(d x) \\
& =\int_{M} \mathcal{L} f(x) \mu(d x) .
\end{aligned}
$$

An invariant measure $\mu$ is called ergodic if for any invariant set $U$, i.e. any set $U \subset M$ satisfying

$$
\mathbb{P}[\omega \in \Omega: \phi(t, p, \omega) \in U, \forall t \geq 0]=1 \quad \text { for all } p \in U,
$$

we have that $\mu(U) \mu\left(U^{c}\right)=0$.

An important result for invariant measures is the following ergodic theorem.

Theorem 2.2: Let $\mu$ be an invariant measure and $f \in L^{1}(\mu)$, then, there is a function $f^{*} \in$ $L^{1}(\mu)$ such that

(i) $f^{*}=\lim _{t \rightarrow \infty} \frac{1}{t} \int_{0}^{t} T_{s} f d s \quad \mu$-almost everywhere (a.e.);

(ii) $\int_{M} f^{*}(x) \mu(d x)=\int_{M} f(x) \mu(d x)$.

Also, when $\mu$ is ergodic and $f \in L^{1}(\mu)$, we have that

$$
f^{*}=\int_{M} f(x) \mu(d x) \quad \mu-\text { a.e. }
$$

For more details about the above result and its proof, see, for example, [11, p. 379]. 


\section{Accessible sets and invariant measures}

In this section, we will present some results which will allow us to characterize accessible sets through transition probabilities, and later by invariant measures.

At first, we give a lemma that is a consequence of the support theorem.

Lemma 3.1: Let $\mathcal{A}(p)$ be the accessible set from $p$ associated to the control system (1) and let $\left\{P_{t}(p, \cdot), t \geq 0\right\}$ be the transition probabilities defined by the SDE (2). Then,

$$
\mathcal{A}(p) \subseteq \bigcup_{t \geq 0} \operatorname{supp}\left(P_{t}(p, \cdot)\right) \subseteq \overline{\mathcal{A}(p)}, \quad \forall p \in M
$$

Proof: To see the first inclusion, we consider a point $q \in \mathcal{A}(p)$. Then, by definition, there will be a control path $p_{u}(s)$ such that $p_{u}(0)=p$ and $p_{u}(t)=q$. Let $B_{q}$ be an open neighbourhood of $q$ and consider the open subset $U_{t}\left(B_{q}\right)$ of the paths space defined by

$$
U_{t}\left(B_{q}\right)=\left\{s \in C([0, \infty), M), s(0)=p, s(t) \in B_{q}\right\}
$$

Since $p_{u}(s) \in U_{t}\left(B_{q}\right)$, we get that $U_{t}\left(B_{q}\right)$ has non-empty intersection with the set of all control paths $\mathcal{C P}_{p}$ starting at $p$, and therefore, by the support theorem, we have that $\mathbb{P}_{p}\left[U_{t}\left(B_{q}\right)\right]>0$. So $P_{t}\left(p, B_{q}\right)>0$, and by the arbitrariness of $B_{q}$, we get that $q \in \sup p\left(P_{t}(p\right.$, $\cdot))$.

For the second inclusion, we assume that $q \in \operatorname{supp}\left(P_{t}(p, \cdot)\right)$. Then, for any open neighbourhood $B_{q}$ of $q$, we have that $P_{t}\left(p, B_{q}\right)>0$. Defining $U_{t}\left(B_{q}\right)$ as above, we get that $\mathbb{P}_{p}\left[U_{t}\left(B_{q}\right)\right]>0$, and therefore by the support theorem, we get that $U_{t}\left(B_{q}\right) \cap \mathcal{C} \mathcal{P}_{p} \neq \emptyset$. Therefore, there is a control path $p_{u}$ such that $p_{u}(0)=p$ and $p_{u}(t) \in B_{q}$. By the arbitrariness of the open neighbourhood $B_{q}$, we get a sequence of points in $\mathcal{A}(p)$ converging to $q$. Thus, $q \in \overline{\mathcal{A}(p)}$.

From this, it follows another result between closure of accessible sets and transition probabilities.

Theorem 3.2: Consider the control system (1). Then, $\overline{\mathcal{A}(p)}=M$ for all $p$ if and only if

$$
\int_{0}^{\infty} P_{t}(p, U) d t>0
$$

for all $p \in M$ and open subsets $U$ of $M$.

Proof: We assume that $\overline{\mathcal{A}(p)}=M$, for all $p \in M$. Then, by Lemma 3.1, for any open set $U$, we have that

$$
\left(\bigcup_{t \geq 0} \operatorname{supp}\left(P_{t}(p, \cdot)\right)\right) \cap U \neq \emptyset .
$$


Thus, since the $P_{t}(p, \cdot)$ are continuous in $t$, we get that there will be a small interval $I=\left(t_{0}\right.$ $\left.-\epsilon, t_{0}+\epsilon\right)$ such that $P_{t}(p, U)>0$, for any $t \in I$. Therefore,

$$
\int_{0}^{\infty} P_{t}(p, U) d t>0,
$$

for all $p \in M$ and open subsets $U$ of $M$.

Now suppose $\overline{\mathcal{A}(p)} \neq M$ for some $p \in M$. Then, for the open set $U=M \backslash \overline{\mathcal{A}(p)}$, we have, by Lemma 3.1, that $P_{t}(p, U)=0$ for all $t \geq 0$, and therefore

$$
\int_{0}^{\infty} P_{t}(p, U) d t=0 .
$$

Let $\mathcal{S}$ be the subset of $M$ given by

$$
\mathcal{S}=\bigcup_{\mu \in \mathcal{M}, \text { ergodic }} \operatorname{supp}(\mu)=\bigcup_{\mu \in \mathcal{M}} \operatorname{supp}(\mu),
$$

where $\mathcal{M}$ is the set of invariant measures under $\mathcal{L}$.

Theorem 3.3: The following assertions are equivalent:

(i) $\overline{\mathcal{A}(p)}=M$ for all $p \in M$.

(ii) Every invariant measure $\mu$ of the diffusion given by Equation (2) satisfies $\operatorname{supp}(\mu)=$ $M$.

Moreover, if (i) or (ii) is satisfied, then

$$
\left\{f \in C^{2}(\mathcal{S}) \cap \mathcal{D}(\mathcal{L}), \mathcal{L} f=0 \text { in } \mathcal{S}\right\}=\{f=\text { const. }\} .
$$

Proof: $(i) \Rightarrow($ ii) Assume that $\overline{\mathcal{A}(p)}=M$ for all $p \in M$. If $p \in \sup p(\mu)$, then

$$
\operatorname{supp}\left(P_{t}(p, \cdot) \subseteq \operatorname{supp}(\mu)\right.
$$

for all $t \geq 0$. In fact, if $\delta_{p}$ is the Dirac $\delta$-measure supported at $p$, we have that $\operatorname{supp}\left(\delta_{p}\right) \subseteq \operatorname{supp}(\mu)$. Thus,

$$
\operatorname{supp}\left(T_{t}^{*} \delta_{p}\right) \subset \operatorname{supp}\left(T_{t}^{*} \mu\right)=\operatorname{supp}(\mu)
$$

We observe that $P_{t}(p, \cdot)=T_{t}^{*} \delta_{p}$. In fact, for every continuous function $f$

$$
\begin{aligned}
T_{t} f(p) & =\int_{M} T_{t} f(y) \delta_{p}(d y) \\
& =\int_{M} f(y) T_{t}^{*} \delta_{p}(d y) .
\end{aligned}
$$

Then, $\mathcal{A}(p) \subseteq \operatorname{supp}(\mu)$ by Lemma 3.1. Thus, $M=\operatorname{supp}(\mu)$. 
(ii) $\Rightarrow$ (i) For the converse, we assume that for any invariant measure $\mu$, we have that $\operatorname{supp}(\mu)=M$. Define the measures

$$
\mu_{t}=\frac{1}{t} \int_{0}^{t} T_{r}^{*} \delta_{p} d r, \quad t \geq 0 .
$$

Clearly for each $t, \mu_{t}$ is a probability measure. We also observe that

$$
T_{s}^{*} \mu_{t}=\mu_{t}-\frac{s}{t} \mu_{s}+\frac{1}{t} \int_{t}^{t+s} T_{r}^{*} \delta_{p} d r .
$$

Since $M$ is compact, there is a sequence $t_{k} \uparrow \infty$ and a probability measure $v$ such that $\mu_{t_{k}} \rightarrow v$. Therefore, $v$ is invariant since

$$
\begin{aligned}
T_{s}^{*} v & =\lim _{k \rightarrow \infty} T_{s}^{*} \mu_{t_{k}} \\
& =\lim _{k \rightarrow \infty} \mu_{t_{k}}=v .
\end{aligned}
$$

Thus,

$$
M=\operatorname{supp}(v) \subseteq \bigcup_{k} \operatorname{supp}\left(P_{t_{k}}(p, \cdot)\right)
$$

and $M \subset \overline{\mathcal{A}(p)}$.

To prove the last statement, we assume that $\overline{\mathcal{A}(p)}=M$ for all $p \in \mathcal{S}$. It is clear that if $f$ is a constant function, then $C^{2}(\mathcal{S}) \cap \mathcal{D}(\mathcal{L})$ and $\mathcal{L} f=0$. On the other side, if $f \in C^{2}(\mathcal{S}) \cap$ $\mathcal{D}(\mathcal{L})$ is a function such that $\mathcal{L} f=0$, then,

$$
\left.f\right|_{\mathcal{S}}=f^{*}=\lim _{t \rightarrow \infty} \frac{1}{t} \int_{0}^{t} T_{s} f d s
$$

and, given an ergodic measure $\mu$, Theorem 2.2 implies that

$$
\left.f\right|_{\operatorname{supp}(\mu)}=\int_{M} f(x) \mu(d x) \quad \mu \text { - a.e. }
$$

Since $\operatorname{supp}(\mu)=\overline{\mathcal{A}(p)}$, for some $p \in M$, we get $\operatorname{supp}(\mu)=M$. Therefore, the function $f$ is constant on the whole manifold $M$.

Remark 3.4: To conclude this section, we observe that it is not true that

$$
\left\{f \in C^{2}(\mathcal{S}) \cap \mathcal{D}(\mathcal{L}), \mathcal{L} f=0 \text { in } \mathcal{S}\right\}=\{f=\text { const. }\}
$$

implies that the leaves are dense. For example, consider the suspension of a linear Anosov diffeomorphism $\phi$ on the two torus as a one-dimensional foliation, this is $M=T^{2} \times[0,1] /$ $\sim$, where $(x, 0) \sim(\phi(x), 1)$ with the foliation defined by the trajectory of the suspension flow determined by $\partial_{t}$. We observe that this foliation has no dense leaves; in fact, they are compact. Any continuous function $f$ such that

$$
\mathcal{L} f=\frac{1}{2} \partial_{t}^{2} f=0
$$


is constant on each of the leaves of the support of any invariant measure (since $M$ is compact, there are invariant measures). This implies that $f([x, t])=k(x)$ for a continuous function $k$ : $T^{2} \rightarrow T^{2}$. But then,

$$
k(x)=f([x, 0])=f([\phi(x), 1])=k(\phi(x)) .
$$

Since $\phi$ is Anosov, $k$ must be constant on $T^{2}$. Therefore, $f$ is constant in $M$.

\section{Conditions in the context of foliation}

Let $F$ be a family of vector fields in $M$ and let $\mathcal{D}_{\text {Lie }(F)}$ the associated distribution to the Lie algebra of $F$. We know that $\mathcal{D}_{\text {Lie(F) }}$ define a foliation in $M$ with its integral manifolds determined by the orbits of $F$.

Using the Nash embedding theorem, it is possible to prove that, for Nlarge enough, there is a family of vector fields $H=\left\{X_{1}, \ldots, X_{N}\right\}$ such that, if $\pi(p): T_{p} M \rightarrow \mathcal{D}_{\text {Lie(F) }}(p) \subset T_{p} M$ is the orthogonal projection and $f$ is a smooth function, then,

$$
\nabla^{D} f(p):=\pi(p)(\nabla f(p))=\sum_{i=1}^{N}\left(X_{i} f\right)(p) X_{i}(p),
$$

and

$$
\left\langle\nabla^{D} f(p), \nabla^{D} f(p)\right\rangle=\sum_{i=1}^{N}\left(X_{i} f\right)^{2}(p)
$$

In fact, consider an isometric embedding of $M$ in $\mathbb{R}^{N}$, via Nash theorem, for $N$ large enough. Let $\left\{e_{1}, \ldots, e_{N}\right\}$ be an orthonormal basis of $\mathbb{R}^{N}$ and consider the vector fields $\left\{\tilde{X}_{1}, \ldots, \tilde{X}_{N}\right\}$ over $M$ defined by

$$
\tilde{X}_{i}(p)=\operatorname{Proj}_{T_{p} M} e_{i}
$$

If $\pi(p): T_{p} M \rightarrow \mathcal{D}_{\operatorname{Lie}(F)}(p) \subset T_{p} M$ is the orthogonal projection, we denote by $X_{i}$ the vector fields given by

$$
X_{i}(p)=\pi(p) \tilde{X}_{i}(p)
$$

Clearly, since $\left\{\tilde{X}_{1}(p), \ldots, \tilde{X}_{N}(p)\right\}$ spans $T_{p} M$, we have that $\left\{X_{1}(p), \ldots, X_{N}(p)\right\}$ spans $\mathcal{D}_{\text {Lie }(F)}(p)$. We observe that each orthonormal basis of $\mathbb{R}^{N}$ will induce a set of vector fields like this. Moreover, if $B_{1}=\left\{e_{1}, \ldots, e_{N}\right\}$ and $B_{2}=\left\{v_{1}, \ldots, v_{N}\right\}$ are two orthonormal bases of $\mathbb{R}^{N}$ and $\left\{X_{1}, \ldots, X_{N}\right\}$ and $\left\{Y_{1}, \ldots, Y_{N}\right\}$ are the vector fields constructed as above for these bases, the orthogonal matrix $O$ for which transforms $B_{1}$ into $B_{2}$ will transform the set of vector fields, this is

$$
Y_{j}=\sum_{i=1}^{N} O_{j i} X_{i}
$$

Then, for every smooth function $f$, there are smooth functions $a_{1}, \ldots, a_{N}$ such that

$$
\nabla^{D} f(p)=\pi_{p}(\nabla f(p))=\sum_{i=1}^{N} a_{i}(p) X_{i}(p) .
$$


Now consider a basis $B_{2}$ of $\mathbb{R}^{N}$ such that for $k>n=\operatorname{dim}(M)$ we have that $v_{k}$ is orthogonal to $T_{p} M$. Then,

$$
\begin{aligned}
X_{j} f(p) & =<\nabla^{D} f(p), X_{j}(p)> \\
& =\sum_{i=1}^{N} a_{i}(p)<X_{i}(p), X_{j}(p)> \\
& =\sum_{i, \alpha, \beta=1}^{N} a_{i}(p) O_{i \alpha} O_{j \beta}<Y_{\alpha}(p), Y_{\beta}(p)> \\
& =\sum_{i, \alpha=1}^{N} a_{i}(p)\left(O O^{T}\right)_{i j}=a_{j}(p) .
\end{aligned}
$$

Thus,

$$
\nabla^{D} f(p)=\pi_{p}(\nabla f(p))=\sum_{i=1}^{N}\left(X_{i} f\right)(p) X_{i}(p)
$$

Similarly,

$$
\begin{aligned}
\left\langle\nabla^{D} f(p), \nabla^{D} f(p)\right\rangle & =\sum_{i, j=1}^{N}\left(X_{i} f\right)(p)\left(X_{j} f\right)(p)<X_{i}(p), X_{j}(p)> \\
& =\sum_{i, j, \alpha, \beta=1}^{N}\left(X_{i} f\right)(p)\left(X_{j} f\right)(p) O_{i \alpha} O_{j \beta}<Y_{\alpha}(p), Y_{\beta}(p)> \\
& =\sum_{i, j, \alpha, \beta=1}^{N}\left(X_{i} f\right)(p)\left(X_{j} f\right)(p)\left(O O^{T}\right)_{i j} \\
& =\sum_{i=1}^{N}\left(X_{i} f\right)^{2}(p) .
\end{aligned}
$$

With the vector fields $\left\{X_{i}, \ldots, X_{N}\right\}$ defined as above, we construct an SDE

$$
d x_{t}=\sum_{i=1}^{N} X_{i}\left(x_{t}\right) \circ d B_{t}^{i}, \quad x_{0}=p,
$$

whose solution is a diffusion with infinitesimal generator given by

$$
\Delta=\frac{1}{2} \sum_{i=1}^{N} X_{i}^{2}
$$

Lemma 4.1: The support of the transition probabilities associated to the SDE (5) are the leaves of the foliation given by $\mathcal{D}_{\operatorname{Lie}(F)}$.

Proof: For a point $p \in M$, consider the leaf $L_{p}$ with the metric induced from $M$. It is simple to see that the Levi-Civita connection $\nabla^{D}$ on $L_{p}$ is related to the Levi-Civita connection $\nabla$ 
on $M$ by

$$
\nabla_{X}^{D} Y(p)=\pi(p)\left(\nabla_{X} Y\right)(p)
$$

for every vector field $X, Y \in \mathcal{D}_{\text {Lie(F) }}$. Now consider an orthonormal basis $\left\{v_{1}, \ldots, v_{k}\right\}$ of $\left(\mathcal{D}_{\text {Lie }(F)}\right)_{p}$ and extending, using again the argument of the isometric embedding, to an orthonormal basis $\left\{v_{1}, \ldots, v_{N}\right\}$ of $\mathbb{R}^{N}$. Let $\left\{e_{1}, \ldots, e_{N}\right\}$ be the basis that defines the vector fields $X_{i}^{\prime} s$ and denote by $Y_{i}$ the ones induced by the $v_{i}^{\prime}$. Again, there is an orthogonal matrix $O$ such that

$$
Y_{i}=\sum_{i=1}^{N} O_{i j} X_{j}
$$

Using a similar argument to the one given in the book of Hsu [12, pp. 77-78] for the Laplace operator on $M$, we get

$$
\begin{aligned}
\Delta_{L_{p}}(f) & \left.=\sum_{i=1}^{k}<\nabla_{v_{i}}^{D} \nabla^{D} f, v_{i}\right\rangle \\
& =\sum_{i=1}^{N} Y_{i}^{2} f(p) \\
& =\sum_{i, j, l=1}^{N} O_{i j} O_{i l} X_{i} X_{l} f(p) \\
& =\sum_{i=1}^{N}\left(X_{i}^{2} f\right)(p) .
\end{aligned}
$$

Therefore, the diffusion defined by (5) is a Brownian motion on the leaf of the associated foliation. That is, if $W^{p}$ is the solution starting at $p$, then $W^{p}$ is a Brownian motion on $L_{p}$ and the transition probability measures $P_{t}(p, \cdot)$ are absolutely continuous to the Lebesgue measure of the leaf. In fact, they will have the form

$$
P_{t}(p, U)=\int_{U} q_{t}^{L_{p}}(x, y) d y
$$

where $q_{t}^{L_{p}}(x, y)$ is the heat kernel on the leaf $L_{p}$.

Once we have that the transition probabilities of the process defined by Equation (5) are supported in the whole leaf, we can use this process to characterize the density of the accessible sets. We do this in the following theorem, where we also give another proof of Krener's theorem [13].

Theorem 4.2: The following assertions are equivalent:

(i) The leaves $\mathcal{A}(p)$ of the foliation defined by $\mathcal{D}_{\text {Lie(F) }}$ are dense in $M$.

(ii) Every invariant measure $\mu$ of the $\operatorname{SDE}(5)$ satisfies $\operatorname{supp}(\mu)=M$. 
Moreover, if (i) or (ii) is satisfied, then

$$
\left\{f \in C^{2}(\mathcal{S}) \cap \mathcal{D}(\Delta), \Delta f=0 \text { in } \mathcal{S}\right\}=\{f=\text { const. }\} .
$$

Proof: The assertions follow from Theorem 3.3 applied to the control system associated to Equation (5).

Corollary 4.3: If $\left(\mathcal{D}_{\text {Lie }(F)}\right)_{p}=T_{p} M$, for all $p$ in $M$, then $\mathcal{A}(p)=M$ for all $p \in M$.

Proof: Let $\left(\mathcal{D}_{\operatorname{Lie}(F)}\right)_{p}=T_{p} M$, for all $p$ in $M$. The construction of the vector fields defining Equation (5) implies that its solution is the Brownian motion of the manifold starting at $p .{ }^{1}$ Then, the support of the transition probabilities is the whole manifold. But, from Lemma 4.1, we get that the support of the transition function is the leaves of the associated foliation, which is just one leaf equal to $M$. Therefore, $\mathcal{A}(p)=M$ for all $p \in M$.

Remark 4.4: Theorem 4.2 can be applied in the context of a foliated $(M, \mathcal{F})$ manifold if we replace $\mathcal{D}_{\text {Lie( } F)}$ by the distribution that define the foliation $\mathcal{F}$.

Example 4.5: Consider the torus given by $\mathbb{T}^{2}=([0,1] \times[0,1]) / \sim$ where $(0, y) \sim(1, y)$ $\mathrm{e}(x, 0) \sim(x, 1)$ and let $X$ be the vector field defined by

$$
X=\partial_{x}+3 \cdot \partial_{y} .
$$

The associated SDE is given by

$$
d p_{t}=X\left(p_{t}\right) \circ d B_{t}
$$

for $B_{t}$ a one-dimensional Brownian motion. The infinitesimal generator for the above SDE is

$$
\mathcal{L}=\frac{1}{2}\left(\partial_{x}^{2}+9 \cdot \partial_{y}^{2}+6 \cdot \partial_{x y}\right)
$$

Therefore, the measure $d \mu$ on $\mathbb{T}^{2}$ given by

$$
d \mu=\rho d m=\sin ^{2}(2 \pi(3 x-y)) d x d y
$$

is invariant. In fact, let $\mathcal{L}^{*}$ the adjoint operator of $\mathcal{L}$, namely, in the language of the theory of partial differential equations, the operator defined by the identity

$$
\int_{\mathbb{T}^{2}}(\mathcal{L} f) \rho d m=\int_{\mathbb{T}^{2}} f\left(\mathcal{L}^{*} \rho\right) d m,
$$

for all function $f$ on $\mathbb{T}^{2}$. From this identity and Equation (3), we obtain

$$
\int_{\mathbb{T}^{2}} f\left(\partial_{t} \rho-\mathcal{L}^{*} \rho\right) d m=0,
$$

and, therefore, the well-known Kolmogorov's forward or the Fokker-Planck equation

$$
\partial_{t} \rho-\mathcal{L}^{*} \rho=0
$$


Furthermore, by Green's theorem, it could be shown that for this special infinitesimal generator $\mathcal{L}=\frac{1}{2} X^{2}$, we have $\mathcal{L}=\mathcal{L}^{*}$. Since

$$
-\frac{1}{2}\left(\partial_{x}^{2}+9 \cdot \partial_{y}^{2}+6 \cdot \partial_{x y}\right)(\rho(x, y))=0
$$

we conclude that $\mu$ is an invariant measure. Since it is possible to find a closed saturated set with non-zero measure, it follows that the leaves are not dense.

Example 4.6: Consider again a compact Riemannian manifold $M$ of $\operatorname{dim}(M)=3$ with an orthonormal basis $\{X, Y, H\}$ of $T M$ satisfying

$$
[X, H]=X, \quad[X, Y]=-H, \quad[H, Y]=Y .
$$

Let $\mathcal{F}$ be a foliation induced by $E=\operatorname{span}\{X, H\}$. The diffusion obtained by Equation (5) is the Brownian motion on the leaves. It could be shown by Garnett (see [7, Proposition 5], or [14, Section 3.4]) that this diffusion has just one invariant measure which is the Lebesgue measure. Then, the leaves of this foliation are dense.

\section{Application on compact homogeneous manifold}

In this section, let $G$ be a Lie group and $\Gamma$ be a closed subgroup of $G$ with cofinite volume, i.e. $M \simeq G / \Gamma$ is a compact homogeneous manifold (see, for example, [15]). Let $\mathfrak{h} \subset \mathfrak{g}$ be a Lie subalgebra and consider the distribution $T \mathcal{F}_{g}=\left(L_{g}\right)_{*} \mathfrak{h}$ which defines a foliation $\mathcal{F}$ on M.

Consider now a basis $\mathcal{B}=\left\{\tilde{X}_{1}, \ldots, \tilde{X}_{k}, \tilde{Y}_{1}, \ldots, \tilde{Y}_{r}\right\}$ of $\mathfrak{g}$ such that $\left\{\tilde{X}_{1}, \ldots, \tilde{X}_{k}\right\}$ is a basis of $\mathfrak{h}$ and $\left\{\tilde{Y}_{1}, \cdots, \tilde{Y}_{r}\right\}$ are identified with a basis of $\mathfrak{g} / \mathfrak{h}$. Then, there are constants $\left\{a_{i j}^{l}, b_{i j}^{l}, c_{i j}^{l}, d_{i j}^{l}, e_{i j}^{l}\right\}$ such that

$$
\begin{aligned}
& {\left[\tilde{X}_{i}, \tilde{X}_{j}\right]=\sum_{l} a_{i j}^{l} \tilde{X}_{l},} \\
& {\left[\tilde{X}_{i}, \tilde{Y}_{\alpha}\right]=\sum_{l} b_{i \alpha}^{l} \tilde{X}_{l}+\sum_{\beta} c_{i \alpha}^{\beta} \tilde{Y}_{\beta},} \\
& {\left[\tilde{Y}_{\alpha}, \tilde{Y}_{\beta}\right]=\sum_{l} d_{\alpha \beta}^{l} \tilde{X}_{l}+\sum_{\gamma} e_{\alpha \beta}^{\gamma} \tilde{Y}_{\gamma} .}
\end{aligned}
$$

Moreover, we can choose right invariant vector fields $\left\{X_{1}, \ldots, X_{k}, Y_{1}, \ldots, Y_{r}\right\}$ on $M$, associated to $\mathcal{B}$, in such a way that $T \mathcal{F}=\operatorname{span}\left\{X_{1}, \ldots, X_{k}\right\}$. We observe that the leaves of $\mathcal{F}$ are the control paths associated to $T \mathcal{F}$, and all of them are isometric.

Let $\langle$,$\rangle denote the invariant metric on M$ such that the set of vector fields

$$
\left\{\tilde{X}_{1}, \ldots, \tilde{X}_{k}, \tilde{Y}_{1}, \cdots, \tilde{Y}_{r}\right\}
$$

is an orthonormal set, and let $\Delta_{\mathcal{F}}$ be the Laplace operator on the leaves, given by

$$
\Delta_{\mathcal{F}}(f)=\operatorname{div}(\pi(\nabla f))
$$


where $\pi: T M \rightarrow T \mathcal{F}$ is the orthogonal projection and $\nabla$ is the Levi-Civita connection. Then,

$$
\begin{aligned}
\Delta_{\mathcal{F}}(f) & =\operatorname{div}(\pi(\nabla f)) \\
& =\sum_{i=1}^{k}\left\langle\nabla_{X_{i}}(\pi(\nabla f)), X_{i}\right\rangle+\sum_{j=1}^{r}\left\langle\nabla_{Y_{j}}(\pi(\nabla f)), Y_{j}\right\rangle \\
& =\sum_{i=1}^{k} X_{i}^{2} f-\pi\left(\nabla_{X_{i}} X_{i}\right) f \\
& =\sum_{i=1}^{k} X_{i}^{2} f+\sum_{i, j=1}^{k} a_{i j}^{i} X_{j} f .
\end{aligned}
$$

We observe that the SDE

$$
\begin{aligned}
d g_{t} & =2 \sum_{i, j=1}^{k} a_{i j}^{i} X_{j}\left(g_{t}\right) d t+\sum_{i=1}^{k} X_{i}\left(g_{t}\right) \circ d B_{t}^{i} \\
g_{0} & =g
\end{aligned}
$$

has $\Delta_{\mathcal{F}}$ as its infinitesimal generator. This process is known as the foliated Brownian motion $($ see $[9,7])$ and the transition probabilities satisfy $\operatorname{supp}\left(P_{t}(g, \cdot)\right)=\overline{\mathcal{F}}_{g}$, the closure of the leave through $g$.

We consider the differential forms $\chi$ and $v$ defined by

$$
\chi=X_{1}^{\mathrm{b}} \wedge \cdots \wedge X_{k}^{\mathrm{b}}, \quad v=Y_{1}^{\mathrm{b}} \wedge \cdots \wedge Y_{r}^{\mathrm{b}}
$$

where $Z^{b}$ denotes the 1 -form $Z^{b}(W)=\langle Z, W\rangle$ for every vector field $W$.

Since for the invariant vector fields associated to $\mathcal{B}$ we have

$$
\begin{aligned}
{\left[X_{i}, X_{j}\right] } & =\sum_{l} a_{i j}^{l} X_{l}, \\
{\left[X_{i}, Y_{\alpha}\right] } & =\sum_{l} b_{i \alpha}^{l} X_{l}+\sum_{\beta} c_{i \alpha}^{\beta} Y_{\beta}, \\
{\left[Y_{\alpha}, Y_{\beta}\right] } & =\sum_{l} d_{\alpha \beta}^{l} X_{l}+\sum_{\gamma} e_{\alpha \beta}^{\gamma} Y_{\gamma},
\end{aligned}
$$

we get that

$$
\begin{aligned}
& L_{X_{i}} X_{l}^{\mathrm{b}}=-\sum_{j} a_{i j}^{l} X_{j}^{\mathrm{b}}-\sum_{\alpha} b_{i \alpha}^{l} Y_{\alpha}^{\mathrm{b}}, \\
& L_{X_{i}} Y_{\beta}^{\mathrm{b}}=-\sum_{\alpha} c_{i \alpha}^{\beta} Y_{\alpha}^{\mathrm{b}} .
\end{aligned}
$$


Therefore,

$$
L_{X_{i}}(\chi \wedge v)=-\operatorname{Tr}\left(\operatorname{ad}\left(X_{i}\right)\right)(\chi \wedge v)
$$

Lemma 5.1: Assume that $\operatorname{tr}(\operatorname{ad}(X))=0$ for every $X \in \mathfrak{h}$, then, the measure $\mu$ induced by $\chi \wedge v$ defines a harmonic probability measure. Moreover, the leafwise Laplacian is self-adjoint with respect to $\mu$.

Proof: By the computation,

$$
\begin{aligned}
\int_{M} \Delta_{\mathcal{F}} f d \mu & =\sum_{i=1}^{k} \int_{M} X_{i}^{2} f \mu+\sum_{i, j=1}^{k} a_{i j}^{i} \int_{M} X_{j} f \mu \\
& =\sum_{i=1}^{k} \int_{M} f L_{X_{i}}^{2} \mu+\sum_{i, j=1}^{k} a_{i j}^{i} \int_{M} f L_{X_{j}} \mu .
\end{aligned}
$$

Thus, $\mu$ is invariant for $\Delta_{\mathcal{F}}$. Doing a renormalization by a constant, we can assume that $\mu$ is a harmonic probability measure with full support for the foliation $\mathcal{F}$ (see $[9,7]$ ).

The leafwise Laplacian $\Delta_{\mathcal{F}}$ is self-adjoint, since

$$
\int_{M}\left(h \Delta_{\mathcal{F}} f-f \Delta_{\mathcal{F}} h\right) d \mu=\frac{1}{2} \sum_{i=1}^{k} \int_{M} X_{i}\left(h X_{i} f-f X_{i} h\right) d \mu=0
$$

Now, assume that $\mu_{1}$ is another harmonic probability measure such that its support is a closed saturated set $K \subsetneq M$. The Radon-Nikodym derivative $d \mu_{1} / d \mu=f$ is a leafwise harmonic function on each leaf $L \subset K$; in fact,

$$
\begin{aligned}
\int_{M}\left(\Delta_{\mathcal{F}} f\right)^{2} d \mu & =\int_{M}\left(\Delta_{\mathcal{F}}^{2} f\right) f d \mu \\
& =\int_{M}\left(\Delta_{\mathcal{F}}^{2} f\right) d \mu_{1}=0 .
\end{aligned}
$$

Therefore, $f$ is constant on $\mu$ almost each $L$ (Garnett [7, Thm. 1.b]). Let $L_{0} \subset K$ be a leaf such that $\left.f\right|_{L_{0}} \neq 0$. Let $T$ be an open transversal section to $L_{0}$ and consider the saturated set

$$
B_{T}=\bigcup_{L \in \mathcal{F}, L \cap T \neq \emptyset} L
$$

Each leaf through $T$ will intercept $T$ at a single point $t$. Denote by $L_{t}$ to the leaf such that $T \cap L_{t}=\{t\}$. Let $g_{t} \in G$ be such that $g_{t} \cdot L_{t}=L_{0}$; moreover, the map $L_{g_{t}}: M \rightarrow M$ defined by $L_{g_{t}}(x)=g_{t} \cdot x$ defines an isometry and therefore $L_{t}$ and $L_{0}$ are isometric.

Consider now the global function $F: M \rightarrow \mathbb{R}$ defined by

$$
F(x)=\left\{\begin{array}{cl}
f\left(g_{t} \cdot x\right) & \text { if } x \in L_{t} \subset B_{T} \\
0 & \text { if } \quad x \notin B_{T}
\end{array}\right.
$$


Since

$$
\Delta_{\mathcal{F}} F(x)=\left(\Delta_{\mathcal{F}} f\right)\left(g_{t} \cdot x\right)=0,
$$

we get that $F$ is leafwise harmonic. Thus, the function $F$ is also constant almost every leaf. So, if $f=k>0$, we get

$$
\begin{aligned}
\int_{B_{T}} d \mu_{1} & =\int_{B_{T}} f d \mu \\
& =\int_{T}\left(\int_{L_{t}} F d \chi\right) d v \\
& =k \operatorname{vol}\left(L_{0}\right) v(T) .
\end{aligned}
$$

With the same assumptions at the beginning of this section, and as a consequence of the discussion above, we obtain the following:

Theorem 5.2: Let $\mathfrak{h} \subset \mathfrak{g}$ be a Lie subalgebra such that $\operatorname{tr}(\operatorname{ad}(X))=0$ for each $X \in \mathfrak{h}$ and let $\mathcal{F}$ be the induced foliation on $M$ by $\mathfrak{h}$. If the leaves $\mathcal{F}_{g}$ of $M$ have infinite volume, then they are dense in $M$.

Corollary 5.3: If $\mathfrak{h} \subset \mathfrak{g}$ is an ideal, $\operatorname{tr}_{\mathfrak{h}}$ ad $: \mathfrak{h} \rightarrow \mathbb{R}$ is the trivial map and the leaves $\mathcal{F}_{g}$ have infinite volume, then the leaves are dense in $M$.

\section{Note}

1. In fact, compare the construction of the process given with the construction of the Brownian motion given, for example, in [12, pp. 77-78].

\section{Disclosure statement}

No potential conflict of interest was reported by the authors.

\section{ORCID}

Fabiano B. da Silva @ (ㄴ) http://orcid.org/0000-0002-2217-8518

\section{References}

[1] A. Agrachev and Y.L. Sachkov, Control Theory from the Geometric Viewpoint, Vol. 87, Springer Science \& Business Media, Berlin, New York, 2013.

[2] H. Kunita, Stochastic Flows and Stochastic Differential Equations (Reprint of the 1990 original), Cambridge Studies in Advanced Mathematics, Vol. 24, Cambridge University Press, Cambridge, New York, 1997.

[3] F. Colonius and W. Kliemann, The Dynamics of Control, Springer Science \& Business Media, Boston, 2012.

[4] P. Stefan, Accessible sets, orbits, and foliations with singularities, Proc. Lond. Math. Soc. 3 (1974), pp. 699-713.

[5] N. Ikeda and S. Watanabe, Stochastic Differential Equations and Diffusion Processes, 2nd ed., North-Holland, Kodansha, 1989. 
[6] W. Kliemann, Recurrence and invariant measures for degenerate diffusions, Ann. Probab., 15 (1987), pp. 690-707.

[7] L. Garnett, Foliations, the ergodic theorem and Brownian motion, J. Funct. Anal. 51 (1983), pp. 285-311.

[8] A. Candel, The harmonic measures of Lucy Garnett, Adv. Math. 176 (2003), pp. 187-247.

[9] P.J. Catuogno, D.S. Ledesma, and P.R. Ruffino, Foliated stochastic calculus: Harmonic measures. Trans. Amer. Math. Soc. 368 (2016), pp. 563-579.

[10] K.D. Elworthy, Stochastic Differential Equations on Manifolds, Vol. 70, Cambridge University Press, Cambridge; New York, 1982.

[11] K. Yosida, Functional Analysis, 6th ed., Grundlehren der Math. Wissenschaften 123, SpringerVerlag, Berlin, 1968.

[12] E.P. Hsu, Stochastic Analysis on Manifolds, Vol. 38, American Mathematical Society, Providence, R. I., 2002.

[13] A.J. Krener, A generalization of Chow's theorem and the bang-bang theorem to nonlinear control problems, SIAM J. Control 12 (1974), pp. 43-52.

[14] B. Deroin and V. Kleptsyn, Random conformal dynamical systems, Geom. Funct. Anal. 17 (2007), pp. 1043-1105.

[15] H. Abbaspour and M. Moskowitz, Basic Lie Theory, World Scientific Publishing Co. Inc., Hackensack, N. J., 2007. 